\title{
Transpapillary Gallbladder Drainage for Acute Cholecystitis: Two Stents or Not Two Stents
}

\author{
Jad AbiMansour and Ryan Law \\ Division of Gastroenterology and Hepatology, Mayo Clinic, Rochester, MN, USA
}

See "Endoscopic Transpapillary Gallbladder Drainage for Acute Cholecystitis using Two Gallbladder Stents (Dual Gallbladder Stenting)” by Zain A Sobani, Sergio A. Sánchez-Luna, Tarun Rustagi, on page 899-902.

In patients with acute cholecystitis who are deemed unfit or high-risk for surgery, percutaneous cholecystostomy has traditionally been the gold standard for management. This approach subjects the patient to a cumbersome external drain that requires routine exchange with defined risks of migration, infection, and permanent percutaneous fistula formation after the drain is removed. Furthermore, percutaneous drainage decreases quality of life and may be a destination therapy for many patients. ${ }^{1,2}$ Given these limitations, an endoscopic approach to gallbladder drainage is preferable. Since the early 1980s, endoscopic transpapillary gallbladder drainage (ETPGBD) has remained the procedure of choice in patients with a patent cystic duct and in institutions with available endoscopic expertise. ${ }^{3}$ However, available data on ETPGBD has been lacking due to the limited procedural availability and the breadth of technical variations. The vast majority of reports describing ETPGBD employ a single stent and describe clinical success rates varying widely from 64 to $100 \%$. A recent meta-analysis by Mohan et al. included 1,223 patients who underwent ETPGBD with a pooled clinical success rate of $83 \%{ }^{5}$

In the current issue of Clinical Endoscopy, Sobani et al. ${ }^{6}$ re-

Received: August 4, 2021 Revised: August 4, 2021

Accepted: August 7, 2021

Correspondence: Ryan Law

Division of Gastroenterology and Hepatology, Mayo Clinic, 200 First Street SW, Rochester, MN 55895, USA

Tel: +1-507-774-2174, Fax: +1-507-266-0350, E-mail: Law.Ryan@mayo.edu ORCID: https://orcid.org/0000-0001-7048-9268

(cc) This is an Open Access article distributed under the terms of the Creative Commons Attribution Non-Commercial License (http://creativecommons.org/ licenses/by-nc/3.0) which permits unrestricted non-commercial use, distribution, and reproduction in any medium, provided the original work is properly cited. ported the retrospective outcomes of 21 patients with acute cholecystitis who underwent ETPGBD using a dual stent technique. This technique was used to improve clinical success. They placed two $22 \mathrm{~cm}$ double pigtail ureteral stents during the index procedure or sequentially with the second stent placed 4 to 6 weeks later. The approach was determined by endoscopist discretion. Notably, there was no comparator group. Furthermore, the criteria for requiring a second transcystic stent were incompletely defined. This small patient cohort demonstrated good technical success (100\%) with clinical recurrence of acute cholecystitis occurring in only one patient (5\%) during a mean follow-up period of greater than one year. Nevertheless, clinical success was defined as the resolution of acute cholecystitis, and this definition is subject to interpretation.

The outcomes of this study using dual transcystic gallbladder stents are consistent with prior data from the Mayo Clinic. Storm et al. described a similar approach in their cohort of 51 patients who underwent either single or dual stent therapy as destination treatment for acute cholecystitis. ${ }^{7}$ When comparing the two cohorts, durable clinical success was achieved in $90 \%$ of patients with a single stent and in $100 \%$ of patients with a dual stent, with two patients having recurrent cholecystitis at a mean of 70 days. Statistical significance was achieved when comparing the number of repeat endoscopic retrograde cholangiopancreatography (ERCP) favoring dual stent therapy ( 1 vs. 1.3 ERCPs; $p=0.045$ ). However, the available data in totality is inadequate to promote dual stent therapy as the new standard of care.

Gallbladder stenting is protective against acute cholecystitis through multiple mechanisms, including draining through a 
capillary-like wicking effect, decompression of the gallbladder, minimization of transmural forces to limit local ischemia, and prevention of stone impaction in the cystic duct. ${ }^{8}$ However, it is unclear how these mechanisms and the clinical efficacy of endoscopic transpapillary therapy are influenced by procedural variables such as stent caliber, number of stents, or other stent properties. The current study utilized 6 to $8.5 \mathrm{Fr}$ double pigtail ureteral stents made of a soft polyurethane material. The stent caliber was similar to that commonly used at most centers for transpapillary drainage, with diameters as small as $3 \mathrm{Fr}$ and as large as $10 \mathrm{Fr} .{ }^{9} 10$ There is no clear consensus on optimal stent size, but utilizing the Goldilocks principle 5-7 Fr plastic pigtails are most common. Smaller diameter stents are used for wicking with almost no role for drainage via the stent lumen, while larger caliber stents provide drainage through the stent lumen. Larger caliber stents occlude more of the cystic duct lumen, thus limiting the wicking effect and nearly guaranteeing the need for stent exchange once the stent occludes. In our opinion, the use of dual transcystic stents can be considered. However, two stents of a small caliber (5 Fr) made of rigid polyethylene may be preferable for two main reasons: (1) two small-caliber stents may adequately splint the spiral valves of the cystic duct without occluding the duct lumen; and (2) dual soft polyurethane stents are prone to collapse, thus limiting drainage via the stent lumen while occluding the cystic duct.

Recently available data on dual transpapillary gallbladder stents are intriguing, at the very least, and certainly require further exploration in a randomized trial format. The 95\% success rate reported by Sobani et al. ${ }^{6}$ is promising. However, it is difficult to generalize this data since the sample consisted of only 21 patients, and there was a lack of a comparator group. Ultimately, a multidisciplinary approach is required in patients with acute cholecystitis who are not surgical candidates. In these patients, optimal therapy is dependent on numerous factors. While ETPGBD is an established endoscopic option, the results of this study suggest that dual stenting is technically feasible and provides a relatively durable response. Currently, there are no high-quality data suggesting that a particular combination of stent number, size, or type is superior to others. It is likely that a multitude of endoscopic approaches can be successful in this scenario; in the absence of rigorous prospective studies, endoscopists should familiarize themselves with the various techniques and apply them as dictated by pa- tient factors, technical feasibility, and center experience when pursuing transpapillary gallbladder drainage.

Conflicts of Interest

Ryan Law is a consultant for Medtronic and ConMed. The other author has no potential conflicts of interest.

Funding

None.

Author Contributions

Writing-original draft: Jad AbiMansour, Ryan Law

Writing-review\&editing: JA, RL

ORCID

Jad AbiMansour:

Ryan Law:

https://orcid.org/0000-0001-8776-588X https://orcid.org/0000-0001-7048-9268

\section{REFERENCES}

1. de Mestral C, Gomez D, Haas B, Zagorski B, Rotstein OD, Nathens AB Cholecystostomy: a bridge to hospital discharge but not delayed cholecystectomy. J Trauma Acute Care Surg 2013;74:175-179; discussion 179180.

2. McKay A, Abulfaraj M, Lipschitz J. Short-and long-term outcomes following percutaneous cholecystostomy for acute cholecystitis in high-risk patients. Surg Endosc 2012;26:1343-1351.

3. Kozarek RA. Selective cannulation of the cystic duct at time of ERCP. J Clin Gastroenterol 1984;6:37-40.

4. Sobani ZA, Ling C, Rustagi T. Endoscopic transpapillary gallbladder drainage for acute cholecystitis. Dig Dis Sci 2021;66:1425-1435.

5. Mohan BP, Khan SR, Trakroo S, et al. Endoscopic ultrasound-guided gallbladder drainage, transpapillary drainage, or percutaneous drainage in high risk acute cholecystitis patients: a systematic review and comparative meta-analysis. Endoscopy 2020;52:96-106.

6. Sobani ZA, Sánchez-Luna SA, Rustagi T. Endoscopic transpapillary gallbladder drainage for acute cholecystitis using two gallbladder stents (dual gallbladder stenting). Clin Endosc 2021;54:899-902.

7. Storm AC, Vargas EJ, Chin JY, et al. Transpapillary gallbladder stent placement for long-term therapy of acute cholecystitis. Gastrointest Endosc 2021;S0016-5107(21)00264-9.

8. Schlenker C, Trotter JF, Shah RJ, et al. Endoscopic gallbladder stent placement for treatment of symptomatic cholelithiasis in patients with end-stage liver disease. Am J Gastroenterol 2006;101:278-283.

9. Yoshida M, Kato A, Hayashi K, et al. Novel technique for intraductal cholangioscopy-assisted biliary drainage with over-the-wire microcatheter manipulation. Endoscopy 2019;51:E398-E399.

10. Mutignani M, Iacopini F, Perri V, et al. Endoscopic gallbladder drainage for acute cholecystitis: technical and clinical results. Endoscopy 2009;41:539-546. 